\title{
A SARS-CoV-SPECIFIC PROTEIN ENHANCES VIRULENCE OF AN ATTENUATED STRAIN OF MOUSE HEPATITIS VIRUS
}

\author{
Lecia Pewe, Haixia Zhou, Jason Netland, Chandra Tangadu, Heidi \\ Olivares, Lei Shi, Dwight Look, Thomas Gallagher, and Stanley Perlman*
}

\section{INTRODUCTION}

Infection of humans with SARS-CoV (severe acute respiratory syndrome-coronavirus) resulted in a severe respiratory syndrome with substantial mortality, especially in the elderly., ${ }^{1,2}$ Although SARS-CoV is the first human coronavirus observed to cause serious disease, coronaviruses were associated with severe disease in several animal species. Pigs infected with transmissible gastroenteritis virus and felines infected with feline infectious peritonitis virus are well-described examples of severe coronavirusinduced diseases. The most intensely studied animal infection is that caused by mouse hepatitis virus (MHV). MHV is a well-nown cause of acute and chronic neurological infections. ${ }^{3}$ It is best-nown for its ability to induce an immune-mediated disease in mice that resembles the human disease, multiple sclerosis. The JHM strain is used in many of these studies and the JHM J2.2-V-1 attenuated variant is particularly useful for studies of chronic demyelination. ${ }^{4}$ Infection with JHM J2.2-V-1 results in infection of oligodendrocytes with minimal infection of neurons, and consequently, low mortality.

SARS-CoV is tentatively classified as a group 2 coronavirus, distantly related to other group 2 coronaviruses such as MHV. Like MHV, it is believed to cause immunemediated disease ${ }^{1}$ SARS-CoV replicates in macrophages and dendritic cells, although the infection is abortive. ${ }^{5-7}$ Replication in these cells results in induction of several cytokines/chemokines including IL-6, IL-8, CCL2, and CXCL10 but not type I interferons, which may contribute to a dysregulated immune response. Elucidation of the role of the host immune response would best be determined in an animal model of SARS, especially because SARS has not recurred to a significant extent in humans since 2003. Several animal models for SARS exist, but they do not reproducibly develop clinical

\footnotetext{
*Lecia Pewe, Haixia Zhou, Jason Netland, Lei Shi, Dwight Look, Stanley Perlman, University of Iowa, Iowa City, Iowa 52242. Chandra Tangadu, Heidi Olivares, Thomas Gallagher, Loyola University Stritch School of Medicine, Maywood, Illinois 60153.
} 
disease. In particular, mice can be infected with SARS-CoV but remain asymptomatic (reviewed in Ref. 1).

Like other coronaviruses, SARS-CoV encodes several nonstructural proteins at the 3 ' end of the genome, flanking the structural genes. One of these "nonstructural proteins" is now known to be a structural protein (ORF3a protein) but the function of the others remains unknown. ${ }^{8}$ In other coronavirus infections, these nonstructural proteins can often be deleted without any effects on growth in tissue culture cells and in some cases, their absence does not seem to affect infection in vivo. ${ }^{9}$ To begin to understand the function of these proteins, we introduced them singly into the genome of JHM J2.2-v-1 and analyzed the effect of the insertion on disease pathogenesis.

\section{MATERIALS AND METHODS}

\subsection{Recombinant Viruses}

Targeted recombination was used to develop recombinant JHM J2.2-V-1 expressing individual SARS-CoV nonstructural proteins. ${ }^{10}$ The development of these viruses is described in more detail elsewhere. ${ }^{11}$ Briefly, PCR products corresponding to nucleotides 25268-26092 (ORF3a), 25689-26153 (ORF3b), 27074-27265 (ORF6), 27273-27641 (ORF7a), 27638-27772 (ORF7b), and 27779-27898 (ORF8) were generated by RT-PCR using RNA harvested from cells infected with the Urbani strain of SARS-CoV (GenBank accession number AY278741, kindly provided by Dr. Tom Ksiazek, Centers for Disease Control, Atlanta, GA). Each product was tagged at the $\mathrm{C}$ terminus with the influenza hemagglutinin epitope for ease of detection. These products were inserted into gene 4 of a J2.2-V-1 shuttle vector, which, in turn, was transcribed. The resulting RNA transcript was transfected into cells previously infected with fMHV-JHM, an MHV chimera expressing the feline surface glycoprotein. Recombinant viruses were selected on murine cells. All isolates were sequenced prior to use in animals and 2 isolates of each virus were used in all studies, to control for the introduction of spurious mutations during the process of recombination.

\subsection{Immunofluorescence Assays}

SARS-CoV proteins were detected using anti-HA murine antibody (Covance, Berkeley, CA, mAb HA.11), biotinylated goat anti-mouse ab (Jackson Immunoresearch) and streptavidin-Cy3 (Jackson Immunoresearch).

\section{RESULTS}

\subsection{Development of Recombinant J2.2-V-1 Expressing SARS-CoV Nonstructural Proteins}

We inserted ORFs 3a, 3b, 6, 7a, 7b, and 8 into the genome of the attenuated $\mathrm{J} 2.2-\mathrm{V}-1$ strain of JHM and tagged each gene with the sequence encoding an influenza hemagglutinin tag to facilitate detection (Figure 1). We could detect all of the inserted proteins by Western blot analysis or IFA (Figure 1, Ref. 11). 

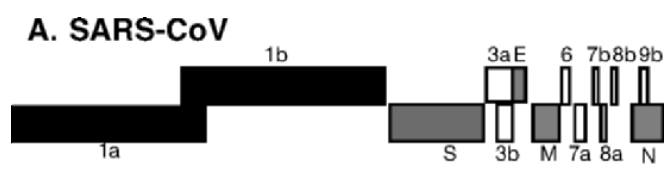

\section{B. MHV}

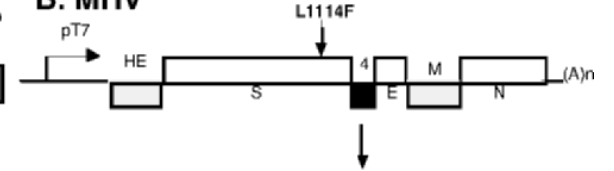

C.
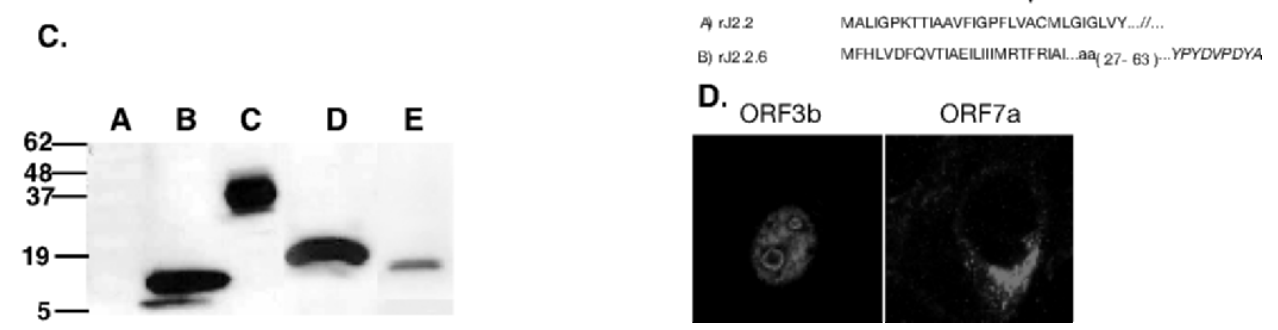

D.

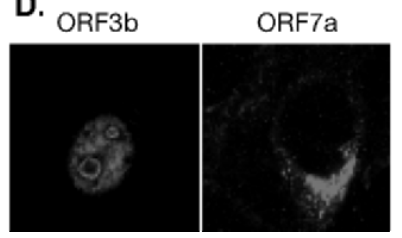

Figure 1. Expression of SARS-CoV nonstructural ORFs in rJ2.2-infected cells. (A). The genome of SARS$\mathrm{CoV}$, with ORFs and structural proteins (gray) is shown. (B). SARS-CoV nonstructural proteins were introduced into $\mathrm{rJ} 2.2$ by targeted recombination (shown for $\mathrm{rJ} 2.2 .6$ ). (C). The products of ORF3a, $6,7 \mathrm{~b}$, and 8 were detected by Western blot analysis using anti-HA antibody. Lanes: A-rJ2.2, B-rJ2.2.6, C-rJ2.2.3a, DrJ2.2.7b, E-rJ2.2.8. (D). All of the inserted proteins were detected by IFA using anti-HA antibody. The products of ORF $3 b, 7 a$ are shown in the figure. Note that protein $3 b$ was localized to the nucleus. No staining was detected in cells infected with rJ2.2 (WT).

\subsection{Infection with Virus Expressing the ORF6 Protein Results in Enhanced Mortality and Clinical Disease}

Next we inoculated mice with recombinant virus and monitored them for survival, clinical signs, and weight loss. Mice inoculated with most recombinant viruses developed a disease very similar to that observed in animals infected with wild-type virus. However, infection with virus expressing the ORF6 protein (rJ2.2.6) caused a fatal disease in mice (Figure 2). In other experiments, we showed that infection with a recombinant virus encoding a mutated form of ORF6 (rJ2.2.6 ${ }^{\mathrm{KO}}$ ) so that the ORF6 RNA was present but no protein expressed did not result in a lethal infection. The presence of the ORF6 protein resulted in higher titers of infectious virus in the CNS than did infection with wild-type virus. ${ }^{11}$ However, these differences were statistically significant only at late times p.i. We confirmed these results by showing that viral RNA levels were also higher in the CNS of mice infected with $\mathrm{rJ} 2.2 .6$ than with $\mathrm{rJ} 2.2 .6^{\mathrm{KO}}$. $^{11}$

\subsection{Infection with rJ2.2.6 Results in Enhanced Growth in Tissue Culture Cells}

These results suggest that ORF6 protein enhances virus growth. To determine whether this also occurs in tissue culture cells, we infected L929 cells with rJ2.2, rJ2.2.6, or $\mathrm{rJ} 2.2 .6^{\mathrm{KO}} \cdot \mathrm{rJ} 2.2 .6^{\mathrm{KO}}$ grew to approximately 1 log higher titers than the other 2 viruses, consistent with the in vivo results. ${ }^{11}$ 
A.

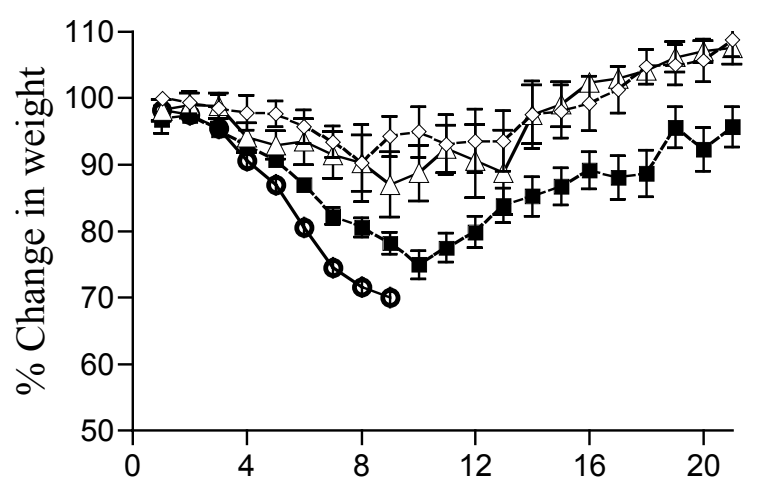

B.

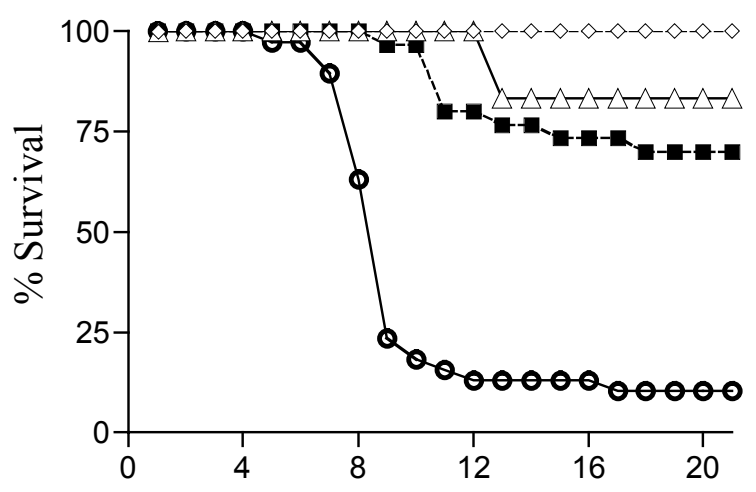

Day p.i

Figure 2. Mortality and morbidity in mice infected with $\mathrm{rJ} 2.2 .6, \mathrm{rJ} 2.2 .6^{\mathrm{KO}}$, and $\mathrm{rJ} 2.2 .8$. Mice infected with $\mathrm{rJ} 2.2$ (triangles), rJ2.2.6 (open circles), rJ2.2.6 ${ }^{\mathrm{KO}}$ (squares), or $\mathrm{rJ} 2.2 .8$ (diamonds) were monitored for (A) mortality and (B) weight loss. In panel (A), data for mice infected with rJ2.2.6 are only shown for days $0-9$ p.i., because only $23 \%$ survived past this time.

\section{4. rJ2.2.6 Does Not Induce Type I Interferon (IFN) or Modulate IFN Sensitivity}

One possibility is that the ORF6 protein affected IFN induction or sensitivity. MHV has been reported not to induce type I interferons ${ }^{12}$ and we confirmed these results. Thus, we were unable to examine whether ORF6 has an additional role in suppressing IFN induction. However, we were able to examine whether rJ2.2.6 affected IFN sensitivity. As shown in Figure 3, cells infected with $\mathrm{rJ} 2.2 .6$ or $\mathrm{rJ} 2.2 .6^{\mathrm{KO}}$ both exhibited similar sensitivities to treatment with IFN- $\beta$. Notably, at all time points, higher virus titers were detected in cells infected with rJ2.2.6 (in the presence or absence or IFN) when compared with their counterparts infected with $\mathrm{rJ} 2.2 .6^{\mathrm{KO}}$. Thus, the presence of ORF6 protein did not affect IFN induction or signaling. 


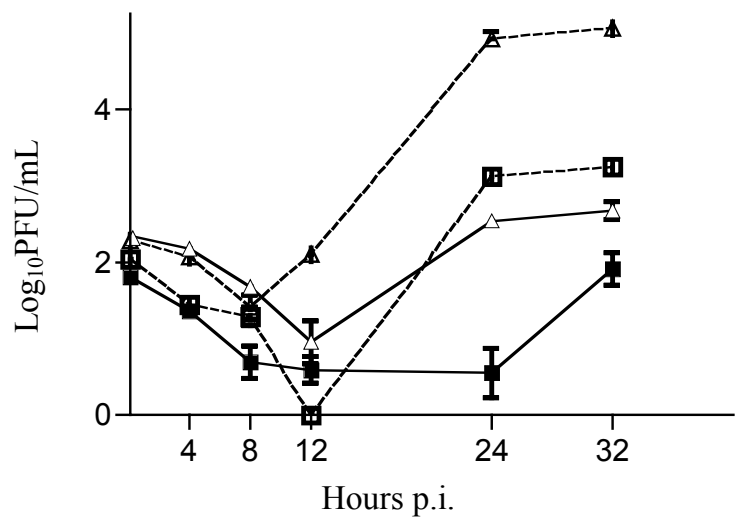

Figure 3. Sensitivity of cells infected with $\mathrm{rJ} 2.2 .6$ and $\mathrm{rJ} 2.2 .6^{\mathrm{KO}}$ to exogenous IFN- $\beta$. Triplicate samples of L929 cells were untreated or treated with $1000 \mathrm{U}$ IFN-B 16 hours prior to infection with $\mathrm{rJ} 2.2 .6$ or $\mathrm{rJ} 2.2 .6^{\mathrm{KO}}$ at $0.1 \mathrm{pfu} / \mathrm{cell}$. IFN- $\beta$ was also present post infection. Cells were harvested at the indicated time points and virus titers determined by plaque assay on HeLa cells expressing the MHV receptor. Open triangle, rJ2.2.6; closed triangle, rJ2.2.6 + IFN; open square, rJ2.2.6 ${ }^{\mathrm{KO}}$; closed square, rJ2.2.6 ${ }^{\mathrm{KO}}+\mathrm{IFN}$.

\subsection{The ORF6 Protein Co-localizes with Membranes}

In additional experiments, we analyzed the localization of the ORF6 protein within infected cells. The primary structure of the ORF6 predicts that it is membrane associated. However, it is not likely to be a transmembrane protein because stretches of hydrophobic residues are interspersed with basic or acidic residues. We performed co-localization experiments using antibodies to the $\mathrm{J} 2.2 \mathrm{~N}, \mathrm{~S}$, and $\mathrm{M}$ proteins and to the endoplasmic reticulum marker, BiP. Our results showed that the ORF6 protein localized to some extent with all the proteins, although co-localization was most evident with the $\mathrm{M}$ protein. We also showed that the ORF6 protein partitioned with membrane fractions after treatment with TX-114. ${ }^{11}$

\section{DISCUSSION}

Our results show that nonstructural proteins from SARS-CoV can function in the context of a murine coronavirus. MHV does not infect lab personnel, thereby circumventing one of the difficulties of working with SARS-CoV. Also, for the first time, we demonstrate a phenotype for a coronavirus nonstructural protein. It is not surprising that a protein from the SARS-CoV can function in the context of a heterologous coronavirus infection. SARS-CoV most likely spread to the human population from an exotic animal species such as palm civet cats or raccoon dogs. ${ }^{2}$ Also, the ORF6 protein is detected in all human and palm civet cat isolates of the SARS-CoV and did not mutate in passage through humans.

Our results suggest that the ORF6 protein enhanced virus growth in tissue culture cells and to a lesser extent, in the infected mouse. However, the presence of the ORF6 protein conferred lethality to an attenuated infection. One explanation for this apparent 
discrepancy is that the ORF6 protein preferentially has an effect in specific cells such as macrophages or dendritic cells, with consequent immune dysregulation. MHV and SARS-CoV are both known to replicate in both cell types, although SARS-CoV, causes an abortive infection. ${ }^{5-7}$ Immune dysregulation is postulated to contribute to SARS pathogenesis. $^{2}$

Our tentative conclusion is that the ORF6 protein directly increases the efficiency of virus replication, assembly, or spread because viral titers, RNA, and protein levels are all increased in cells infected with $\mathrm{rJ} 2.2 .6^{11}$ The protein is not present in virions, showing that it does not have a direct effect on virus infectivity. ${ }^{11}$ The ORF6 protein is broadly distributed throughout the cell, coincident with sites of virus replication (co-localization with the $\mathrm{N}$ protein) and virus assembly (co-localization with the $\mathrm{M}$ protein). Future work will be directed at determining how exactly the presence of this protein enhances virus replication.

This work was supported by a grant from the N.I.H. (PO1 AI606699).

\section{REFERENCES}

1. J. S. Peiris, Y. Guan, and K. Y. Yuen, Severe acute respiratory syndrome, Nat. Med. 10, S88-97 (2004).

2. J. S. Peiris, K. Y. Yuen, A. D. Osterhaus, and K. Stohr, The severe acute respiratory syndrome, N. Engl. J. Med. 349, 2431-2441 (2003).

3. S. Perlman, Pathogenesis of coronavirus-induced infections: Review of pathological and immunological aspects, Adv. Exp. Med. Biol. 440, 503-513 (1998).

4. J. O. Fleming, M. D. Trousdale, F. El-Zaatari, S. A. Stohlman, and L. P. Weiner, Pathogenicity of antigenic variants of murine coronavirus JHM selected with monoclonal antibodies, J. Virol. 58, 869-875 (1986).

5. H. K. Law, et al., Chemokine up-regulation in SARS-coronavirus-infected, monocyte-derived human dendritic cells, Blood 106, 2366-2374 (2005).

6. C. Y. Cheung, et al., Cytokine responses in severe acute respiratory syndrome coronavirus-infected macrophages in vitro: possible relevance to pathogenesis, J. Virol. 79, 7819-7826 (2005).

7. C. T. Tseng, L. A. Perrone, H. Zhu, S. Makino, and C. J. Peters, Severe acute respiratory syndrome and the innate immune responses: Modulation of effector cell function without productive infection, J. Immunol. 174, 7977-7985 (2005).

8. N. Ito, et al., Severe acute respiratory syndrome coronavirus 3 a protein is a viral structural protein, J. Virol. 79, 3182-3186 (2005)

9. E. Ontiveros, L. Kuo, P. S. Masters, and S. Perlman, Inactivation of expression of gene 4 of mouse hepatitis virus strain JHM does not affect virulence in the murine CNS, Virology 290, 230-238 (2001).

10. L. Kuo, G. J. Godeke, M. J. Raamsman, P. S. Masters, and P. J. Rottier, Retargeting of coronavirus by substitution of the spike glycoprotein ectodomain: crossing the host cell species barrier, J. Virol. 74, 13931406 (2000).

11. L. Pewe, et al., A severe acute respiratory syndrome-associated coronavirus-specific protein enhances virulence of an attenuated murine coronavirus, J. Virol. 79, 11335-11342 (2005).

12. L. E. Garlinghouse, Jr., A. L. Smith, and T. Holford, The biological relationship of mouse hepatitis virus (MHV) strains and interferon: in vitro induction and sensitivities, Arch. Virol. 82, 19-29 (1984). 